\title{
Urinary urgency outcomes after propiverine treatment for an overactive bladder: the 'Propiverine study on overactive bladder including urgency data'
}

\author{
Kyu-Sung Lee ${ }^{1}$, Hye Won Lee ${ }^{1}$, Myung-Soo Choo², Jae-Seung Paick ${ }^{3}$, \\ Jeong Gu Lee ${ }^{4}$, Ju Tae Seo ${ }^{5}$, Jeong Zoo Lee ${ }^{6}$, Young-Suk Lee', Hana Yoon ${ }^{7}$, \\ Choal Hee Park ${ }^{8}$, Yong-Gil Na ${ }^{9}$, Young Beom Jeong ${ }^{10}$, Jong Bouk Lee ${ }^{11}$ and \\ Won Hee Park ${ }^{12}$ \\ ${ }^{1}$ Department of Urology, Samsung Medical Center, Sungkyunkwan University School of Medicine, ${ }^{2}$ University of \\ Ulsan College of Medicine, ${ }^{3}$ Seoul National University College of Medicine, ${ }^{4}$ Korea University College of Medicine, \\ ${ }^{5}$ Kwandong University College of Medicine, ${ }^{7}$ Ewha Womans University School of Medicine, and ${ }^{11}$ National Medical \\ Center, Seoul, ${ }^{6}$ Pusan National University College of Medicine, Pusan, ${ }^{8}$ Keimyung University School of Medicine, \\ Daegu, ${ }^{9}$ Chungnam National University College of Medicine, Daejeon, ${ }^{10}$ Chonbuk National University Medical \\ School, Jeonju, and ${ }^{12}$ Inha University College of Medicine, Incheon, Korea \\ Accepted for publication 12 August 2009
}

Study Type - Therapy (RCT) Level of Evidence $1 b$

\section{OBJECTIVE}

To investigate the effects of a daily regimen of propiverine $20 \mathrm{mg}$ in patients with an overactive bladder (OAB), focused on improving urgency, as the clinical efficacy of treatment for $\mathrm{OAB}$ should be measured in terms of urgency, the cornerstone symptom of $O A B$.

\section{PATIENTS AND METHODS}

Eligible patients aged $\geq 18$ years with symptoms of $O A B$ were enrolled in this multicentre, prospective, parallel, doubleblind, placebo-controlled trial. Of 264 patients (mean age 52.2 years), 221 who had efficacy data available from baseline and at least one on-treatment visit with $>75 \%$ compliance with medication were analysed (142 in the propiverine group and 79 in the placebo group). All patients were randomized to receive a placebo or $20 \mathrm{mg}$ propiverine once daily in a 12-week study. They completed a 3-day voiding diary before visits during the study period, including the severity of urgency associated with every voiding, using the Indevus Urgency Severity Scale and the Urgency Perception Score. The patients' overall self-evaluation of treatment benefits at the end of the study, and safety data, were also collected.

RESULTS

The daily urgency episodes reduced significantly from baseline to 12 weeks on propiverine treatment, compared with placebo $(-46.0 \%$ vs $-31.3 \%, P=0.005)$. Secondary endpoints, including sum of urgency severity per $24 \mathrm{~h}$, urgency severity per void, and daytime voiding frequency, were also improved significantly in the propiverine group. Overall, of those patients treated with propiverine, 38.7\% rated their treatment as providing 'much benefit', compared with $15.2 \%$ of the placebo group $(P=0.025)$. Adverse events reported by 32 $(22.5 \%)$ and $10(12.7 \%)$ patients in the propiverine and placebo group were all tolerable. However, this is a short-term study using only one fixed regimen.

\section{CONCLUSIONS}

Propiverine $20 \mathrm{mg}$ once-daily could be an effective treatment for patients with $O A B$, by improving urgency.

\section{KEYWORDS}

overactive bladder, urinary urgency, propiverine hydrochloride, randomized trial

\section{INTRODUCTION}

Overactive bladder $(O A B)$ is defined as 'urgency, with or without urge incontinence, usually with frequency and nocturia' in the absence of local pathological or endocrine factors [1]. In the EPIC study using the 2002 ICS definition, the overall prevalence of $O A B$ was $11.8 \%$ (10.8\% in men and $12.8 \%$ in women) and increased with age [2]. Urgency is defined as 'the complaint of a sudden compelling desire to pass urine that is difficult to defer' and an abnormal sensation that is distinctly different from the normal physiological feeling of 'urge to void' that occurs during typical bladder-filling cycles [3].
Because up to half of patients with $O A B$ have urgency with no incontinence, and because urgency is the most bothersome symptom that drives behavioural adaptations such as frequent voiding because of the very fear of urgency, this is the cornerstone symptom of $O A B$ that indicates the diagnosis of $O A B$ [4-6]. 
Although any effective treatment for $\mathrm{OAB}$ must reduce the patient's sense of urgency, the precise mechanisms of how urgency is perceived and the underlying causes remain to be fully elucidated, and its subjective nature makes it difficult to measure. Therefore, the clinical efficacy of $O A B$ treatment was traditionally measured in terms of objective surrogate variables instead of urgency itself, e.g. change in urinary frequency, incontinent episodes and number of pads, and nonvoiding detrusor contractions on urodynamic study, which could be measured easily and quantifiably $[7,8]$. Recently, several methods that measure urgency have been developed and used in clinical practice [9-11]. A systematic review and meta-analysis of the efficacy, tolerability and safety of licensed antimuscarinic treatments in $O A B$ analysing 73 randomized, placebo-controlled trials showed that active treatments were more effective than placebo in terms of improvement in the number of incontinence episodes per day, voids per day, volume voided per void, and return to continence at endpoint [12]. Although fesoterodine, propiverine, solifenacin and tolterodine improved the number of urgency episodes per day statistically significantly more than placebo (pooled differences in mean change 0.64-1.56 episodes per day) in this meta-analysis, few clinical studies assessing urgency itself as the primary focus for therapeutic intervention with sensitive patient-driven criteria have been conducted until recently.

Propiverine hydrochloride has combined antimuscarinic and calcium-antagonistic actions. Previous trials on the clinical efficacy and safety of propiverine for treating patients with $O A B$ reported improvements in urinary frequency and incontinence, but not in urgency severity and number of urgency episodes $[13,14]$. The aim of the present study was to explore the efficacy of a once-daily regimen of propiverine at $20 \mathrm{mg}$ (immediaterelease formulation) in improving urgency from baseline to 12 weeks of treatment in patients with $O A B$.

\section{PATIENTS AND METHODS}

Eligible men and women aged $\geq 18$ years who had self-reported symptoms of OAB for $\geq 3$ months were enrolled and the study was conducted at 12 study sites in Korea. Patients were required to show an average urinary frequency of $\geq 10$ voids/24 $\mathrm{h}$ and urgency of two or more episodes/24 $\mathrm{h}$ defined as 'moderate to severe' in the Indevus Urgency Severity Scale (IUSS) during the 3-day voiding diary period before randomization [15]. Exclusion criteria were: clinically significant stress urinary incontinence (more than one episode per week); genitourinary conditions that could cause OAB symptoms, such as UTI; and contraindications to the use of antimuscarinic drugs.

Patients who qualified for the study were then randomized at a ratio of 1:2 to placebo or to $20 \mathrm{mg}$ propiverine hydrochloride once daily at the second visit. A randomization list was prepared by a trial-independent statistician using a random permuted-block design. The investigator allocated treatment by assigning patient numbers in a strict consecutive order at the centre. All patients completed a 3-day voiding diary before baseline (visit 1), during treatment week 4 (visit 3) and during treatment week 12 (visit 5). They recorded voiding frequency, voided volume, incontinence episodes and the frequency and severity of urgency associated with voiding, using the IUSS. The study design is illustrated in Fig. 1; it was performed in accordance with the International Conference on Harmonization of Good Clinical Practice guidelines and the principles of the Declaration of Helsinki. The study protocol received ethics committee approval at each study site. Written informed consent was obtained from all patients before screening.

The primary efficacy endpoint was the percentage change from baseline in mean number of urgency events/24 h after 12 weeks of treatment. Secondary efficacy endpoints included the percentage changes from baseline to 12 weeks of treatment in the overall patient perception of urgency, urgency severity/void, the sum of urgencyseverity/24 $h$, daytime and nocturnal voiding frequencyl $24 \mathrm{~h}$. Overall patient perception of urgency was assessed using the Urgency Perception Score (UPS) [9]. Patients were asked to describe their typical experience when he or she felt the desire to urinate. The possible response options were: 1 , 'I am usually not able to hold urine'; 2, 'I am usually able to hold urine until I reach the toilet if I go immediately'; and 3, 'I am usually able to finish what I am doing before going to the toilet'. Finally, the patients' overall self-evaluation of treatment benefit after 12 weeks' treatment was graded as 'no benefit', 'little benefit', or 'much benefit'.
FIG. 1. The study design.

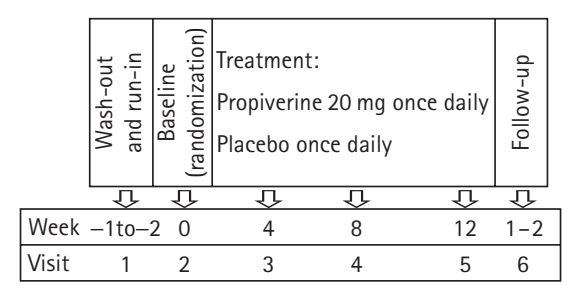

Tolerability and safety data were collected at each visit and at other times if volunteered by the patient. All observed or volunteered adverse events were evaluated by the investigator and the patient in terms of severity. Safety variables, e.g. the incidence and severity of any adverse events, and the incidence and any reason for withdrawal, from baseline to 12 weeks of treatment were recorded.

To test whether propiverine treatment was effective on the primary endpoint, a mean of the primary endpoint in the placebo group and the common SD of the primary end point were assumed to be $12.8 \%$ and $50.0 \%$, respectively. It was also assumed that the target treatment was effective if any mean of the primary endpoint in the treatment group was at $\geq 30 \%$. Therefore, at least 79 patients were required in the placebo group and 158 in the treatment group, assuming 80\% power and $5 \%$ significance level. Assuming a 10\% discontinuation rate, 88 patients were required in the placebo group and 176 in the treatment group.

The primary and secondary endpoints were analysed in the patients who had efficacy data available from the baseline and at least one on-treatment visit with $>75 \%$ compliance with medication. Continuous variables between the groups were compared using two-sample Student's t-tests or MannWhitney U-tests. Changes in ordinal variables between the two groups were compared using the Generalized Estimating Equation (GEE).

Changes in continuous variables and ordinal variables within treatment group were analysed using paired Student's t-tests or Wilcoxon's signed-rank test and GEE, respectively.

\section{RESULTS}

This 12-week, multicentre, prospective, parallel, double-blind, placebo-controlled trial 
FIG. 2. Participant flow; $O D$, once daily.

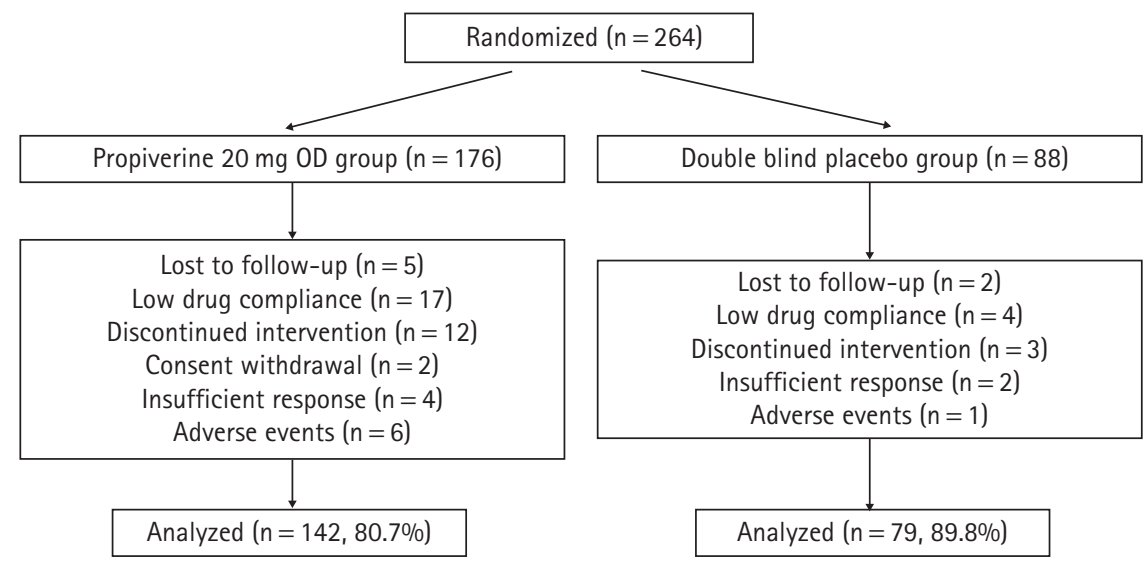

TABLE 1 The baseline demographics and voiding characteristics of the 221 patients, and the secondary efficacy endpoint results from baseline to week 12

\begin{tabular}{|c|c|c|c|}
\hline Mean (SD) or $n(\%)$ variable & Propiverine $20 \mathrm{mg}$ & Placebo & $P$ \\
\hline $\bar{N}(\%)$ patients & $142(64.3)$ & $79(35.8)$ & \\
\hline Age, years & $53.3(11.19)$ & $51.4(12.06)$ & ns \\
\hline Men & $36(25.4)$ & $21(26.6)$ & ns \\
\hline Women & $106(74.6)$ & $58(73.4)$ & ns \\
\hline \multicolumn{4}{|l|}{ Baseline voiding characteristics } \\
\hline No. urgent voids/24 h & $7.4(4.9)$ & $7.6(4.43)$ & ns \\
\hline No. voids/24 h & $12.8(4.2)$ & $13.0(5.6)$ & ns \\
\hline No. diurnal voids/24 h & $11.0(4.1)$ & $11.1(4.9)$ & ns \\
\hline No. nocturnal voids $/ 24 \mathrm{~h}$ & $1.7(1.1)$ & $1.9(1.4)$ & ns \\
\hline Urgency severity score $/ 24 \mathrm{~h}$ & $21.1(11.5)$ & $21.2(8.8)$ & ns \\
\hline Urgency severity score/void & $1.63(0.544)$ & $1.67(0.506)$ & ns \\
\hline Maximum voided volume, $\mathrm{mL}$ & $299.7(195.5)$ & $246.8(79.24)$ & ns \\
\hline Total volume voided/24 h, mL & $1703.5(545.8)$ & 1579.9 (523.8) & ns \\
\hline Mean volume voided/void, $\mathrm{mL}$ & $141.0(52.27)$ & $130.4(47.8)$ & ns \\
\hline \multicolumn{4}{|c|}{ Efficacy, as mean change and mean \% change from baseline to week 12} \\
\hline No. voids/24 h & $-3.56(3.218)$ & $-2.58(4.182)$ & 0.004 \\
\hline$\%$ & $-26.46(17.91)$ & $-17.41(18.87)$ & $<0.001$ \\
\hline No. diurnal voids $/ 24 \mathrm{~h}$ & $-3.04(3.00)$ & $-2.16(3.627)$ & 0.008 \\
\hline$\%$ & $-25.74(18.68)$ & $-16.31(20.39)$ & $<0.001$ \\
\hline No. nocturnal voids/24 h & $-0.52(1.012)$ & $-0.42(1.039)$ & ns \\
\hline$\%$ & $-21.74(62.90)$ & $-9.76(66.93)$ & ns \\
\hline Total sum of urgency severity score $/ 24 \mathrm{~h}$ & $-9.42(9.875)$ & $-6.55(9.523)$ & 0.009 \\
\hline$\%$ & $-41.41(39.94)$ & $-24.63(42.96)$ & 0.001 \\
\hline Urgency severity score/void & $-0.41(0.614)$ & $-0.26(0.645)$ & ns \\
\hline$\%$ & $-23.29(41.18)$ & $-10.98(44.40)$ & 0.028 \\
\hline Maximum voided volume & $1.57(187.2)$ & 14.89 (80.91) & ns \\
\hline$\%$ & $9.45(38.04)$ & $12.67(46.48)$ & ns \\
\hline Total volume voided/24 h & $183.1(555.6)$ & 99.36 (491.90) & ns \\
\hline$\%$ & $3.43(65.67)$ & $1.42(35.74)$ & ns \\
\hline Mean volume voided/void & $35.47(55.52)$ & $19.99(49.53)$ & 0.045 \\
\hline$\%$ & 33.41 (77.89) & $24.33(54.69)$ & ns \\
\hline
\end{tabular}

was conducted between December 2003 and July 2004, and screened 291 patients; 264 with $\mathrm{OAB}$ (mean age 52.2 years, $74 \%$ women) were enrolled, with 176 on propiverine and 88 on placebo after randomization. Forty-three (16.2\%) patients were excluded during the study period and their reasons for withdrawal are shown in Fig. 2. The efficacy analysis (221 patients) included all randomized patients who had efficacy data available from the baseline and at least one on-treatment visit, and who were $>75 \%$ compliant with study medication, including 142 (80.7\%) in the propiverine and 79 (89.8\%) in the placebo group. The treatment and placebo groups were statistically similar for baseline demographics and voiding characteristics (Table 1).

Propiverine treatment resulted in a greater percentage change from baseline in the mean number of urgency episodes/24 $\mathrm{h}$ during the study than among those randomized to placebo (Fig. 3). Compared with the placebo group (reduction of 31\% from baseline), there were statistically significant decreases in the mean number of urgency episodes (reduction of $46 \%$ from baseline) in patients treated with propiverine at week $12(P=0.005)$.

When evaluating secondary efficacy variables after 12 weeks of treatment, propiverine also significantly improved the sum of urgency severity score $/ 24 \mathrm{~h}$ (reductions of $41 \%$ vs $25 \%, P=0.001)$ and urgency severity/void (reductions of $23 \%$ vs $11 \%, P=0.028$ ) as well as the broad range of $24-h$ voiding characteristics, including daytime frequency and voiding frequency/24 $h$, compared with patients receiving placebo, from baseline to endpoint (Table 1). There was a small reduction in the number of nocturnal voids/ $24 \mathrm{~h}$ at week 12, but the differences for propiverine vs placebo were not statistically significant $(P=0.11)$. The other variables, including total voided volume/24 $\mathrm{h}$, mean volume voided, and maximum voided volume, showed no statistically meaningful difference from placebo.

Moreover, propiverine caused an increase in the proportion of patients able to finish tasks before voiding in response to urgency, from $4.9 \%$ to $45.1 \%$, compared to an increase from $3.8 \%$ to $26.6 \%$ for placebo treatment $(P<0.005)$, and a decrease in the proportion of patients unable to hold urine on experiencing urgency, from $26.8 \%$ to $10.6 \%$, vs $24.1 \%$ to $19.1 \%$ in the placebo group 
$(P<0.005)$ (Fig. 4). Overall, a significantly greater proportion of patients perceived benefits with propiverine ( $81 \%$ vs $66 \%$ in the placebo group; $P=0.01$ ) and of those patients treated with propiverine, $38.7 \%$ rated their treatment as providing 'much benefit' compared with $15.2 \%$ of placebo recipients $(P=0.025)$.

Adverse events were reported by 32 (22.5\%) patients in the propiverine and by 10 (12.7\%) patients in the placebo group, and these adverse events were generally mild (Table 2). Visual analogue scale assessment of drymouth symptoms showed that the scale score for the propiverine group after 12 weeks was significantly higher than in the placebo group $(P<0.001)$, although there were no cases of severe dry mouth reported in patients treated with propiverine.

\section{DISCUSSION}

Urgency is the complaint of a sudden compelling desire to pass urine which is difficult to defer, and it must be differentiated from 'urge', which is a normal physiological sensation [10]. Patients with $O A B$ can have both 'urge' and 'urgency' and not every void is associated with urgency. Some plausible but as yet not fully proven theories for the difference in the sensations of urge and urgency suggested that afferent signals conveying urge travel in A $\delta$-fibres, whereas those conveying urgency travel in C-fibres; in addition, urge and urgency can also lead to distinct patterns of activation in the CNS [8]. It is important that patients with $O A B$ can differentiate urge from urgency, and that they can describe urgency severity clearly enough to record it accurately and objectively in voiding diaries [16]. However, there are limitations associated with accurately clarifying the symptoms of urgency to patients, because they can confuse it with a simple urge [5]. Therefore, even though urgency is the cardinal feature of $O A B$, the measure of any successful treatment has traditionally been based on improvements in the reduction in urinary frequency or in incontinent episodes, and urodynamic variables such as detrusor overactivity.

Because urgency might be a central symptom driving urinary frequency, nocturia, and urgency urinary incontinence (UUI), and because it has a significant negative effect on quality of life similar to UUI, the evaluation
FIG. 3. Mean reductions in percentages from baseline in urgency episodes/24 h at weeks 4 and 12 ; although the reduction in the number of urgency episodes from baseline was not statistically significantly different between the groups at week $4(\mathrm{P}=0.81)$, propiverine $20 \mathrm{mg}$ once daily was significantly more effective than placebo in reducing the number of urgency episodes at week $12 .{ }^{*} \mathrm{P}=0.005$.
Week 4

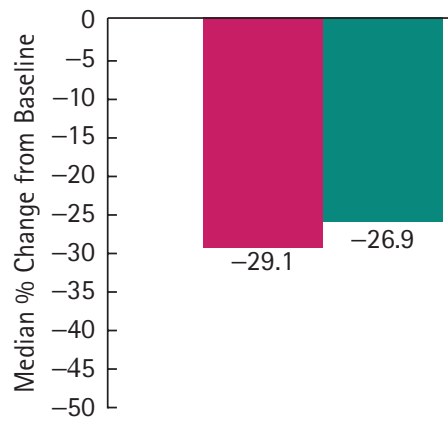

Week 12

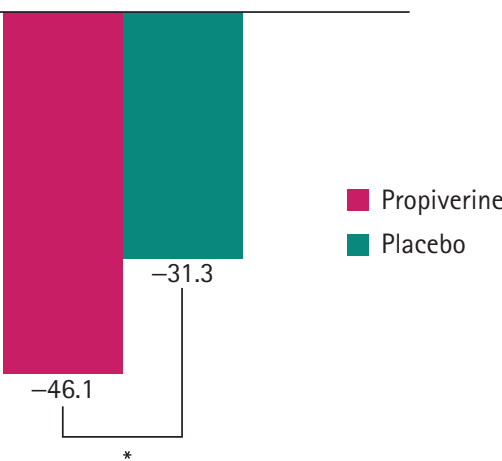

FIG. 4. Improvement in the UPS from baseline to week 12; propiverine caused a increase in the proportion of patients able to finish tasks before voiding in response to urgency, from $4.9 \%$ to $45.1 \%$, compared with an increase from $3.8 \%$ to $26.6 \%$ for placebo treatment $(\mathrm{P}<0.005)$, and a decrease in the proportion of patients unable to hold urine upon experiencing urgency from $26.8 \%$ to $10.6 \%$, compared with a decrease from $24.1 \%$ to $19.1 \%$ in the placebo group $(\mathrm{P}<0.005)$. OD, once daily. ${ }^{*} \mathrm{P}<0.005$; ${ }^{* * P}<0.005$.
Propiverine $20 \mathrm{mg}$ OD

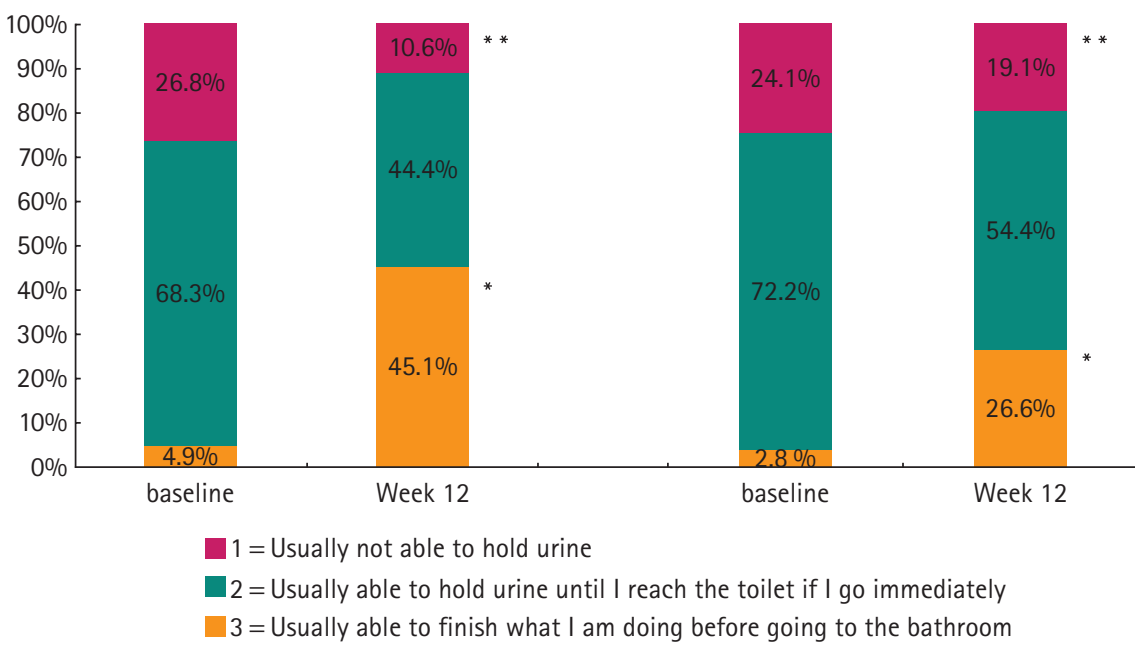

\begin{tabular}{|c|c|c|c|}
\hline Adverse event & Propiverine 20 mg (142) & Placebo (79) & TABLE 2 \\
\hline$\overline{n(\%):}$ & & & Adverse events during the \\
\hline Adverse event rate & $32(22.5)$ & $10(12.7)$ & study period \\
\hline Dry mouth & $14(9.9)$ & $2(2.5)$ & \\
\hline Voiding difficulty & $7(4.9)$ & 0 & \\
\hline Constipation & $5(3.5)$ & 0 & \\
\hline Headache & $1(0.7)$ & $2(2.5)$ & \\
\hline Erectile dysfunction & $1(0.7)$ & $1(1.3)$ & \\
\hline Dizziness & $1(0.7)$ & $2(2.5)$ & \\
\hline
\end{tabular}


and treatment of patients with $O A B$ should focus on ameliorating the sensation of urgency $[11,17]$. We found that a 12-week course of propiverine treatment was statistically significantly more effective than placebo in terms of improving urgencyassociated variables, including the number of urgency episodes/24 $h$, the sum of urgency severity score/24 $\mathrm{h}$ and finally the severity of urgency per voiding episode. In recent study to measure urgency severity as the primary endpoint, solifenacin 5/10 mg was significantly effective in reducing the number of urgency episodes and the extent of urgency bother [18]. However, there was little information about the efficacy of propiverine for treating patients with $O A B$, especially the sensation of urgency. To our knowledge, the present is the first study to evaluate the clinical efficacy of propiverine with a focus on urgency as a primary end point. The efficacy of propiverine for improving urgency was evaluated in terms of the number of urgency episode, urgency severity per voids, total sum of urgency severity score during $24 \mathrm{~h}$ and each patient's perception of urgency. Because urgency drives all the other symptoms of $O A B$, the reduction in urgency could have helped to reduce the frequency of urination, and the discrepancy in terms of daytime and nocturnal improvements in the present study might have been caused by the multifarious aetiology of nocturia.

We used patient-completed voiding diaries and the IUSS and UPS to assess improvements in urgency. Patients were taught to record every void accurately and to note whether each was associated with urgency. Proper education for the concept of urgency was important, because many patients could not understand the difference between the items in the scales of the IUSS. Clinical assessments of urgency seek to measure the number of urgency episodes per day, its degree of severity, and its degree of discomfort to the patient. Several methods have been introduced; patient-completed voiding diaries have been used generally as a primary objective and as a quantifiable tool for measuring changes in the number of urgency episodes during the treatment of $O A B$ in several clinical trials $[19,20]$. In a study analysing the validity of a voiding diary specifically designed to assess the symptoms of $O A B$, the measure of urgency episodes had equal or higher interclass correlation coefficients than those for urinary frequency and incontinence episodes. Thus, the quantitative assessment of urgency with voiding diaries might be a reliable method for measuring this variable [21]. On the other hand, the IUSS and UPS are subjective tools designed to measure urgency severity and each patient's perception. While Chapple et al. [16] stated that urgency is episodic and maximal, some investigators advocate that urgency intensity should be graded, along with the warning time or other validated questionnaires, as under certain circumstances it can be suppressed very easily and voiding can be deferred [22]. The IUSS is a patient-reported outcome measure to quantify the level of urgency associated with each toilet-voiding episode, and consists of four distinct subjective degrees of urgency linked to the level of impairment, as measured by the ability to complete activities [11]. The UPS is scored on a 5-point scale ranging from voiding out of convenience (no urgency, 0 ) to desperate urgency (score of 4) [9]. Both measures have confirmed good validity and inter-test reliability $[9,15]$.

A patient's perception of treatment benefit is a simple scale used to assess this factor. In a study using tolterodine $4 \mathrm{mg}$, the percentage of patients reporting 'much benefit' from treatment at 12 weeks was $43 \%$ in the tolterodine group and $24 \%$ in the placebo group $(P<0.001)$ [23]. Similarly, our study showed that the proportion of patients responding with 'yes' to the question 'have you had any benefit from your treatment?' and rating the outcome as 'much benefit' was significantly higher in the propiverine group. Thus, the objective and subjective improvements of OAB symptoms, including urgency, were reflected in the patients' perceptions of treatment benefits from propiverine treatment.

The present study was a 12-week short-term study using only one fixed regimen of propiverine immediate-release formulation $20 \mathrm{mg}$ once daily, and the patients with $O A B$ were highly selected. With more meaningful data in clinical practice, a long-term study should be conducted based on a flexible-dose regimen according to the improvement in a patient's symptoms. Although suggested possible causes of urgency are spontaneous smooth muscle cell contraction, detrusor micromotions, altered urothelial mediator release acting on smooth muscle or afferent nerves and altered central nervous processing of signals from the bladder, the mechanisms involved in generating the sensation of urgency remain unclear [8]. In addition, urgency remains difficult to define, communicate and measure in a reliable and valid manner that is satisfactory to both clinicians and patients. Further investigations are needed to elucidate the pathophysiology of urgency and the correlations between urgency frequency or severity measurements and other $O A B$ symptoms, or urodynamic variables, to improve the efficacy of treatment for patients with $O A B$ and to develop therapeutic agents selectively suppress nonvoiding detrusor contractions.

\section{ACKNOWLEDGEMENTS}

This study was sponsored by Jeil Pharmaceutical Co., Ltd, Seoul, Korea. Registration identification number; NCT00903045; http://clinicaltrials.gov/ct2/ show/NCT00903045.

\section{CONFLICT OF INTEREST}

Source of Funding: Jeil Pharmaceutical Co., Ltd., Seoul, Korea.

\section{REFERENCES}

1 Milsom I, Abrams P, Cardozo L, Roberts RG, Thuroff J, Wein AJ. How widespread are the symptoms of an overactive bladder and how are they managed? A population-based prevalence study. BJU Int 2001; 87: 760-6

2 Irwin DE, Milsom I, Hunskaar S et al. Population-based survey of urinary incontinence, overactive bladder, and other lower urinary tract symptoms in five countries: results of the EPIC study. Eur Urol 2006; 50: 1306-14

3 Abrams P, Cardozo L, Fall M et al. The standardisation of terminology of lowerurinary tract function: report from the Standardisation Sub-committee of the International Continence Society. Neurourol Urodyn 2002; 21: 167-78

4 Abrams P. Urgency: the key to defining the overactive bladder. BJU Int 2005; 96 (Suppl. 1): 1-3

5 Brubaker L. Urgency: the cornerstone symptom of overactive bladder. Urology 2004; 64: 12-6

6 Coyne KS, Payne C, Bhattacharyya SK et al. The impact of urinary urgency and frequency on health-related quality of life 
in overactive bladder: results from a national community survey. Value Health 2004; 7: 455-63

7 Jabs CF, Stanton SL. Urge incontinence and detrusor instability. Int Urogynecol J Pelvic Floor Dysfunct 2001; 12: 58-68

8 Michel MC, Chapple CR. Basic mechanisms of urgency: preclinical and clinical evidence. Eur Urol 2009; 56: 298307

9 Blaivas JG, Panagopoulos G, Weiss JP, Somaroo C, ChaikinDC. The urgency perception score: validation and testretest. J Uro/ 2007; 177: 199-202

10 Abrams P. Describing bladder storage function: overactive bladder syndrome and detrusor overactivity. Urology 2003; 62: 28-37

11 Starkman JS, Dmochowski RR. Urgency assessment in the evaluation of overactive bladder (OAB). Neurourol Urodyn 2008; 27: 13-21

12 Chapple CR, Khullar V, Gabriel Z, Muston D, Bitoun CE, Weinstein D. The effects of antimuscarinic treatments in overactive bladder: an update of a systematic review and meta-analysis. Eur Urol 2008; 54: 543-62

13 Alloussi S, Goepel M, Richter AE, Eberhardt R, Zwingers T, Brunjes R. [Therapy for overactive detrusor using propiverine]. Urologe A 2005; 44: 3826
14 Madersbacher H, Murtz G. Efficacy, tolerability and safety profile of propiverine in the treatment of the overactive bladder (non-neurogenic and neurogenic). World J Urol 2001; 19: 32435

15 Nixon A, Colman S, Sabounjian L et al. $A$ validated patient reported measure of urinary urgency severity in overactive bladder for use in clinical trials. J Urol 2005; 174: 604-7

16 Chapple CR, Artibani W, Cardozo LD et al. The role of urinary urgency and its measurement in the overactive bladder symptom syndrome: current concepts and future prospects. BJU Int 2005; 95 : 335-40

17 Tubaro A. Defining overactive bladder: epidemiology and burden of disease. Urology 2004; 64: 2-6

18 Cardozo L, Hessdorfer E, Milani R et al. Solifenacin in the treatment of urgency and other symptoms of overactive bladder: results from a randomized, double-blind, placebo-controlled, risingdose trial. BJU Int 2008; 102: 1120-7

19 Madersbacher H, Halaska M, Voigt R, Alloussi S, Hofner K. A placebocontrolled, multicentre study comparing the tolerability and efficacy of propiverine and oxybutynin in patients with urgency and urge incontinence. BJU Int 1999; 84: 646-51
20 Chapple CR, Rechberger T, Al-Shukri S et al. Randomized, double-blind placeboand tolterodine-controlled trial of the once-daily antimuscarinic agent solifenacin in patients with symptomatic overactive bladder. BJU Int 2004; 93 : 303-10

21 Brown JS, McNaughton KS, Wyman JF et al. Measurement characteristics of a voiding diary for use by men and women with overactive bladder. Urology 2003; 61: 802-9

22 Abrams P, Artibani W, Cardozo L, Dmochowski $R$, van Kerrebroeck $P$, Sand P. Reviewing the ICS 2002 terminology report: the ongoing debate. Neurourol Urodyn 2009; 28: 287

23 Freeman R, Hill S, Millard R, Slack $M$, Sutherst J. Reduced perception of urgency in treatment of overactive bladder with extended-release tolterodine. Obstet Gynecol 2003; 102: 605-11

Correspondence: Won Hee Park, 7-206, 3-ga, Sinheung-dong, Jung-gu, Incheon, Postal Code; 400-711 Korea.

e-mail:drwonhee@inha.ac.kr

Abbreviations: $\mathrm{OAB}$, overactive bladder; IUSS, Indevus Urgency Severity Scale; UPS, Urgency Perception Score; GEE, Generalized Estimating Equation; UUI, urgency urinary incontinence. 